\title{
A study of nonlinear waves and resonance in intrusion flows
}

\author{
Michael J. Chen ${ }^{1} \quad$ Lawrence K. Forbes ${ }^{2}$
}

(Received 27 July 2007; revised 13 September 2007)

\begin{abstract}
A stratified intrusion flow is considered in which there are three moving (horizontal) fluid layers and two interfaces. The top and bottom layers move with different speeds and may even move in opposite directions, producing an exchange flow. The middle layer is in motion relative to the outer two, and possesses shear so that the speed in the three-fluid system is continuous when the interfaces are both unperturbed. The flow configuration supports the propagation of periodic waves. A linearized analysis for small wave amplitudes is presented. This is compared to some nonlinear periodic solutions found numerically using a Fourier technique. Such solutions permit nonlinear resonances between the various solution modes and these have been computed extensively.
\end{abstract}

See http://anziamj.austms.org.au/ojs/index.php/ANZIAMJ/article/view/328 for this article, (c) Austral. Mathematical Soc. 2007. Published October 7, 2007. ISSN 1446-8735 


\section{Contents}

1 Introduction

C52

2 Model and governing equations

C53

3 Linearized solution

C56

4 Nonlinear solution

C59

5 Results

C61

6 Conclusion

C66

References

C66

\section{Introduction}

An intrusion flow is approximated by three horizontal fluid layers, each of constant density and in motion. Such flows occur in the atmosphere, for instance, or in runoff flowing into reservoirs. Layered flows of this type are known to support the propagation of periodic, steady waves. The case of a current intruding into a stationary fluid has been considered by Forbes et al. [1], who obtained a number of geometrically limiting solutions. Similar three layer problems have been studied by Michallet and Dias [3] and Rusås and Grue [5], who found that resonant effects were present when two modes of solution were available for a particular set of speed parameters at near integer multiple wavelengths. The focus of this work is the study of these effects for the present problem. There is a possibility that the wave profiles will become multivalued, as was seen in the two layer flows of Pullin and Grimshaw [4] and Hocking and Forbes [2] as well as by Rusås and Grue [5], and this possibility will be accounted for. 
The model is formulated in Section 2; a linearized solution for small amplitudes is presented in Section 3. The nonlinear solution technique is outlined in Section 4, with Section 5 giving an array of numerical solutions.

\section{Model and governing equations}

The flow to be considered consists of three horizontal fluid layers. Throughout the paper these are denoted with subscripts 1, 2 and 3 for the top, middle and bottom layers respectively. Each layer is of constant density $\rho_{i}$, $i=1,2,3$, with $\rho_{1}<\rho_{2}<\rho_{3}$. Two interfaces are present on the upper and lower boundaries of the middle layer, labelled $y=\eta_{U}$ and $y=\eta_{L}$. There is a constant horizontal background flow in the two outer layers, with current speeds $c_{1}$ and $c_{3}$. The background flow in Layer 2 possesses shear so that its speed matches the outer two layers when the interfaces are flat. The fluids are assumed to be incompressible and inviscid; the shear in the middle layer leaves only the two outer layers flowing irrotationally.

By introducing non-dimensional variables the system may be shown to be dependent on the four dimensionless parameters

$$
F_{1}=\frac{c_{1}}{\sqrt{g h}}, \quad F_{3}=\frac{c_{3}}{\sqrt{g h}}, \quad D_{1}=\frac{\rho_{1}}{\rho_{2}}, \quad D_{3}=\frac{\rho_{3}}{\rho_{2}} .
$$

Here the length scale is chosen as $h$, the height of the middle layer. Velocity is scaled with $\sqrt{g h}$, a characteristic speed in the middle layer, to give the Froude numbers $F_{1}$ and $F_{3}$. Density is scaled by the density of the middle layer, $\rho_{2}$, giving two density ratios $D_{1}$ and $D_{3}$. The appropriate form for the linear $y$-dependent background shear flow in the middle layer is

$$
F_{2}(y)=F_{3}+\left(F_{1}-F_{3}\right) y .
$$

This non-dimensional system is illustrated in Figure 1. 


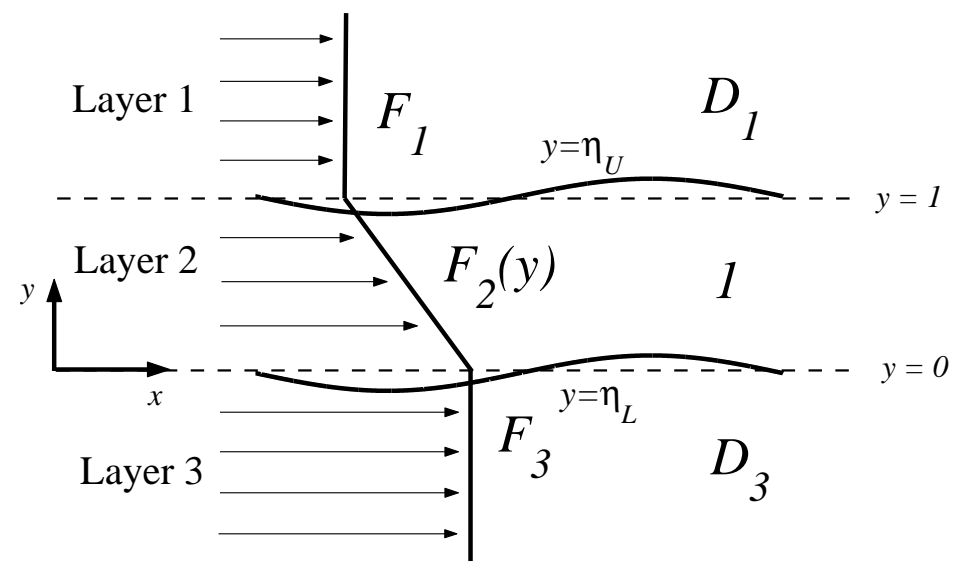

FiguRE 1: A diagram of the flow configuration in non-dimensional variables.

We define fluid velocity vectors $\mathbf{q}_{i}=u_{i} \mathbf{i}+v_{i} \mathbf{j}(i=1,2,3)$ in each layer. In Layers 1 and 3 we also define a velocity potential in the usual manner,

$$
\begin{aligned}
\phi_{i} & =F_{i} x+\Phi_{i}, \\
\mathbf{q}_{i} & =\left(F_{i}+\frac{\partial \Phi_{i}}{\partial x}\right) \mathbf{i}+\frac{\partial \Phi_{i}}{\partial y} \mathbf{j} \quad(i=1,3) .
\end{aligned}
$$

In Layer 2 this is complicated by the presence of shear; no overall velocity potential exists, but we define the velocity vector $\mathbf{q}_{2}$ as

$$
\mathbf{q}_{2}=\left(F_{3}+\left(F_{1}-F_{3}\right) y+\frac{\partial \Phi_{2}}{\partial x}\right) \mathbf{i}+\frac{\partial \Phi_{2}}{\partial y} \mathbf{j},
$$

where $\Phi_{2}$ is a velocity potential for the irrotational component of the flow in Layer 2. In addition, a streamfunction $\psi_{2}$ for Layer 2 is introduced. This must have the form

$$
\psi_{2}(x, y)=F_{3} y+\frac{1}{2}\left(F_{1}-F_{3}\right) y^{2}+\Psi_{2}(x, y),
$$

where the irrotational part of the streamfunction $\Psi_{2}$ is related to $\Phi_{2}$ via the Cauchy-Riemann equations. 
Conservation of mass requires that the velocity potentials satisfy Laplace's equation in each of the three unknown fluid regions

$$
\begin{aligned}
& \nabla^{2} \phi_{1}=0 \text { in } \eta_{U}<y<\infty, \\
& \nabla^{2} \Phi_{2}=0 \text { in } \eta_{L}<y<\eta_{U}, \\
& \text { and } \nabla^{2} \phi_{3}=0 \text { in }-\infty<y<\eta_{L} \text {. }
\end{aligned}
$$

The problem is highly nonlinear as the shape of the regions (and of the two interfaces) is not known beforehand.

Several boundary conditions are specified on each interface. On the upper interface there are two kinematic conditions which require that neither Fluid 1 or Fluid 2 can cross the interface,

$$
v_{i}=u_{i} \frac{\partial \eta_{U}}{\partial x} \quad(i=1,2) \quad \text { on } y=\eta_{U} .
$$

Similarly, on the lower interface we require that

$$
v_{i}=u_{i} \frac{\partial \eta_{L}}{\partial x} \quad(i=2,3) \quad \text { on } y=\eta_{L} .
$$

By equating pressure in Fluid 1 and Fluid 2 at the upper interface (via Bernoulli's equation) we obtain the dynamic condition

$$
\begin{aligned}
& \frac{1}{2} D_{1} F_{1}^{2}-\frac{1}{2} D_{1}\left(u_{1}^{2}+v_{1}^{2}\right)-D_{1}\left(\eta_{U}-1\right) \\
= & \frac{1}{2} F_{3}^{2}-\frac{1}{2}\left(u_{2}^{2}+v_{2}^{2}\right)+\left(F_{1}-F_{3}\right) \psi_{2}-\left(\eta_{U}-1\right) \quad \text { on } y=\eta_{U},
\end{aligned}
$$

and there is likewise the dynamic condition for the lower interface

$$
\begin{aligned}
& \frac{1}{2} F_{3}^{2}-\frac{1}{2}\left(u_{2}^{2}+v_{2}^{2}\right)+\left(F_{1}-F_{3}\right) \psi_{2}-\eta_{L} \\
= & \frac{1}{2} D_{3} F_{3}^{2}-\frac{1}{2} D_{3}\left(u_{3}^{2}+v_{3}^{2}\right)-D_{3} \eta_{L} \quad \text { on } y=\eta_{L},
\end{aligned}
$$


which equates pressure in Fluids 2 and 3 there. Far from the middle layer, flow conditions are uniform, and therefore

$$
\begin{aligned}
& \phi_{1} \rightarrow F_{1} x \text { as } y \rightarrow+\infty, \\
& \phi_{3} \rightarrow F_{3} x \text { as } y \rightarrow-\infty,
\end{aligned}
$$

as Fluids 1 and 3 are of infinite vertical extent. Periodic solutions to equations (9)-(12) are sought for $\phi_{1}, \Phi_{2}, \phi_{3}, \eta_{U}$ and $\eta_{L}$. The assumption of periodicity leads to the introduction of a new parameter, the dimensionless wavenumber $k$. This is now needed, in addition to the dimensionless Froude numbers and density ratios (1), to specify a solution completely.

\section{$3 \quad$ Linearized solution}

A linearized solution to the governing equations (6)-(12) is now presented. We make a perturbation of small amplitude about uniform flow with flat interfaces to obtain a dispersion relation between the parameters. The appropriate perturbed expansions for the velocity potentials are

$$
\Phi_{i}(x, y)=\epsilon \Phi_{i 1}+\mathcal{O}\left(\epsilon^{2}\right) \quad(i=1,2,3),
$$

where $\epsilon$ is a small constant in the order of the wave's amplitude. We likewise perturb the interfacial profiles about $y=0$ and $y=1$ :

$$
\begin{aligned}
& \eta_{L}(x)=\epsilon H_{L 1}(x)+\mathcal{O}\left(\epsilon^{2}\right), \\
& \eta_{U}(x)=1+\epsilon H_{U 1}(x)+\mathcal{O}\left(\epsilon^{2}\right) .
\end{aligned}
$$

In order to satisfy Laplace's equations (6)-(8) the velocity potentials are chosen to be

$$
\begin{aligned}
& \Phi_{11}(x, y)=a_{11} e^{-k(y-1)} \sin k x, \\
& \Phi_{21}(x, y)=\left(c_{2} \cosh k y+d_{2} \sinh k y\right) \sin k x,
\end{aligned}
$$




$$
\Phi_{31}(x, y)=a_{31} e^{k y} \sin k x
$$

These have period $2 \pi / k$ in $x$ and the appropriate $y$-dependence, with $\Phi_{11}$ and $\Phi_{31}$ decaying to zero as $y \rightarrow \infty$ and $y \rightarrow-\infty$ respectively. The streamfunction (5) for Fluid 2 is likewise linearized to give

$$
\psi_{2}(x, y)=F_{3} y+\frac{1}{2}\left(F_{1}-F_{3}\right) y^{2}+\epsilon \Psi_{21}(x, y)+\mathcal{O}\left(\epsilon^{2}\right)
$$

where $\Psi_{21}$ may be determined from the irrotational velocity potential $\Phi_{21}$ via the Cauchy-Riemann equations. These perturbed forms are now substituted into the kinematic and dynamic boundary conditions (9)-(12) (with terms of order order $\epsilon^{2}$ and higher discarded) to obtain a system of linear algebraic equations. These are solved to obtain the dispersion relation

$$
\begin{aligned}
& \mathcal{D}_{1} k F_{3}^{2}+\mathcal{D}_{3} k F_{1}^{2}+\mathcal{D}_{1} \mathcal{D}_{3} \tanh k+k^{2} F_{1}^{2} F_{3}^{2} \tanh k=0, \\
& \text { where } \mathcal{D}_{1}=D_{1} k F_{1}^{2}-F_{1}\left(F_{1}-F_{3}\right)-\left(1-D_{1}\right), \\
& \text { and } \mathcal{D}_{3}=D_{3} k F_{3}^{2}+F_{3}\left(F_{1}-F_{3}\right)-\left(D_{3}-1\right) .
\end{aligned}
$$

From this relation we establish the co-dependency of the Froude numbers and density ratios on wavenumber so that the governing equations (6)-(12) are satisfied to the first order in $\epsilon$. It can be shown, in the long wavelength limit $k \rightarrow 0$, that the dispersion relation (20) becomes

$$
F_{3}=\frac{\left(1-D_{1}\right)\left(D_{3}-1\right)}{F_{1}\left(D_{3}-D_{1}\right)} .
$$

In addition, for short waves $(k \rightarrow \infty)$, it follows that either $F_{3} \rightarrow 0$ or

$$
F_{3} \approx \frac{1-D_{1}+F_{1}^{2}\left(1-k-k D_{1}\right)}{F_{1}} .
$$

The effect of varying each of the five parameters is impractical to investigate fully; instead we fix the value of the two density ratios and the upper layer Froude number $F_{1}$ at values of physical interest. This leaves (20) with two 


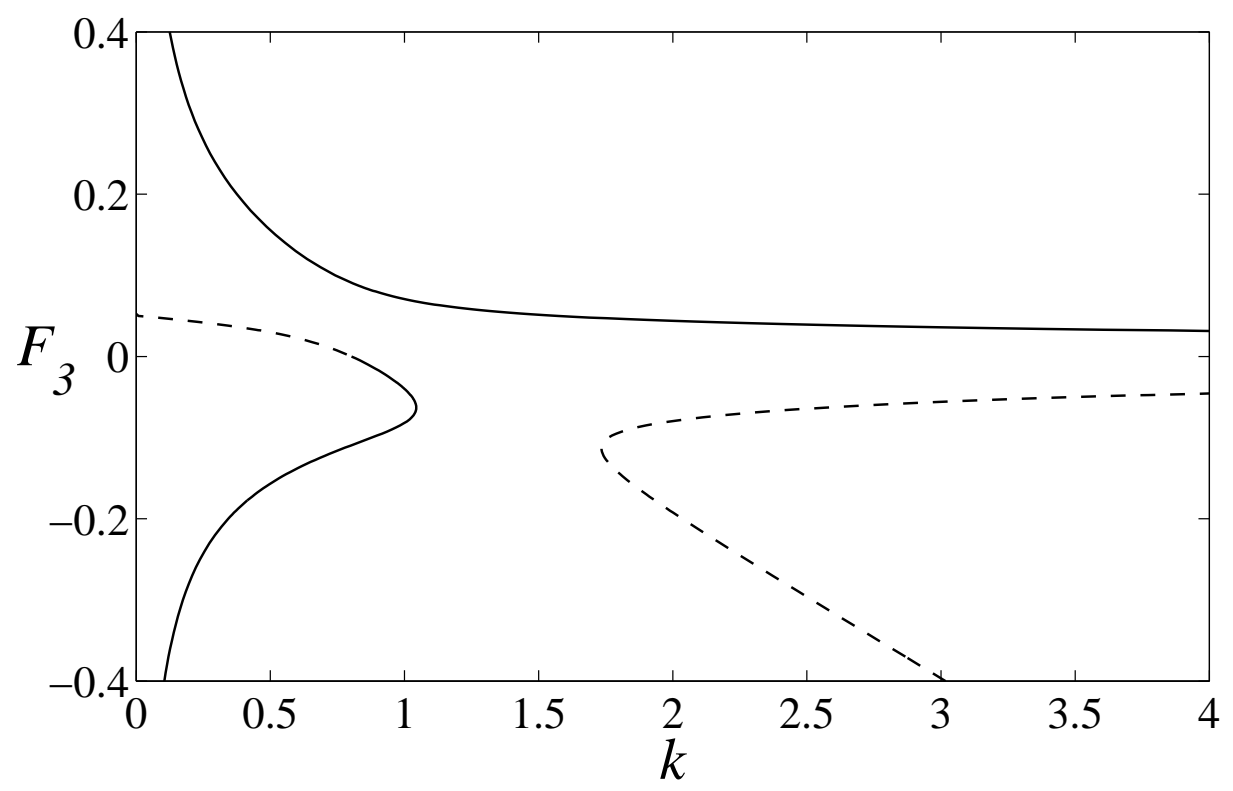

Figure 2: An example solution of the linearized dispersion relation (20) for the lower layer Froude number $F_{3}$ against the wavenumber $k$. 
free parameters, the wavenumber $k$ and lower layer Froude number $F_{3}$. The relationship between these two parameters is investigated by varying one and numerically solving for the other.

An example solution is shown in Figure 2. Here the density ratios have been chosen to be close to unity, with $D_{1}=0.99$ and $D_{3}=1 / 0.99$, and the upper layer Froude number to be $F_{1}=0.1$. In this diagram the solid lines indicate that the interfaces are in phase with each other, and the dashed lines indicate that the interfaces are out of phase. There are three values of Froude number for fixed wavenumber, except for the small interval near $k \approx 1.5$. Likewise, for Froude number less than $F_{3} \approx 5.025 \times 10^{-2}$ there are two possible solutions at different wavenumbers. When one of these wavenumbers is an integer multiple of the other, linear resonance is possible. In a nonlinear regime, where the Froude number is allowed to vary as the waves are computed to finite amplitudes, it may be possible to excite nonlinear resonance effects at near integer multiples.

\section{Nonlinear solution}

Nonlinear solutions to equations (6)-(12) were sought using a numerical procedure. This involves computing Fourier series solutions for the velocity potentials and the interfacial profiles. The profiles are re-parametrized using arc length to allow for the possibility that they become multivalued (as has been seen in similar studies $[4,2,5$, e.g.].

The Fourier series solutions for the velocity potentials must satisfy Laplace's equations (6)-(8) (and decay as in (13)) and are chosen to be

$$
\Phi_{1}=\sum_{n=1}^{N} B_{n} e^{-n k(y-1)} \sin n k x,
$$




$$
\begin{aligned}
\Phi_{2} & =\sum_{n=1}^{N}\left[C_{n} \cosh n k\left(y-\frac{1}{2}\right)+D_{n} \sinh n k\left(y-\frac{1}{2}\right)\right] \sin n k x, \\
\Phi_{3} & =\sum_{n=1}^{N} F_{n} e^{n k y} \sin n k x
\end{aligned}
$$

where these expressions become exact as $N \rightarrow \infty$.

An arclength variable $s$ is now introduced, and the interfacial profiles are re-parametrized in the form $(x(s), y(s))$. By making a change of variables with a new scaled arc length $\xi$,

$$
\xi=\frac{2 \pi s}{L}, \quad 0<\xi \leq 2 \pi,
$$

where $L$ is total arc length over one wave period, the Pythagorean definition of arc length is used to derive an extra condition to be satisfied on each interface:

$$
\left(\frac{d x}{d \xi}\right)_{U, L}^{2}+\left(\frac{d y}{d \xi}\right)_{U, L}^{2}=\frac{L_{U, L}^{2}}{4 \pi^{2}} .
$$

Here the subscripts $U$ and $L$ refer to the upper and lower interfaces, respectively. The two interfaces are represented in terms of their Cartesian coordinates as $(x, y)=\left(x_{L}, \eta_{L}\right)$ for the lower interface and $(x, y)=\left(x_{U}, \eta_{U}\right)$ for the upper interface. These are chosen as $2 \pi$-periodic in $\xi$ with the Fourier series representations:

$$
\begin{aligned}
& {\left[\begin{array}{l}
\eta_{U} \\
\eta_{L}
\end{array}\right]=\left[\begin{array}{c}
1+P_{0} \\
R_{0}
\end{array}\right]+\sum_{n=1}^{N}\left[\begin{array}{l}
P_{n} \\
R_{n}
\end{array}\right] \cos n \xi,} \\
& {\left[\begin{array}{l}
x_{U} \\
x_{L}
\end{array}\right]=\frac{\xi}{k}+\sum_{n=1}^{N}\left[\begin{array}{c}
T_{n} \\
U_{n}
\end{array}\right] \sin n \xi .}
\end{aligned}
$$

Again, these become exact as $N \rightarrow \infty$. In formulating finite amplitude solutions an extra parameter as a measure of wave amplitude is required. 
An appropriate choice is half the peak-to-trough displacement of the upper interface, defined as

$$
2 \mathcal{A}_{U}=\eta_{U}(0)-\eta_{U}(\pi) .
$$

The quantity $\mathcal{A}_{U}$ in equation (29) is generally specified in the numerical method, and $F_{3}$ is obtained as an output.

Each nonlinear solution is specified by the $8 N+2$ Fourier coefficients as well as the lower layer Froude number. These are solved for numerically with wave amplitude held constant. A vector of the $8 N+3$ unknowns is formed for use in a Newton's method routine. Here the error vector is made up of the (slightly rearranged) boundary conditions (9)-(12) multiplied by a Fourier basis function ( $N$ times for each condition) and then integrated over one period. Specifically the kinematic conditions (9)-(10) are multiplied by the odd Fourier basis functions $\sin j \xi(j=1, \ldots, N)$, and integrated, while the dynamic conditions (11)-(12) and the arc length conditions (26) are multiplied by the even Fourier bases $\cos j \xi(j=1, \ldots, N)$, thus giving $8 N$ components of the error vector. The remaining three components are obtained by integrating each of the dynamic conditions without premultiplication and from the wave amplitude condition (29). The trapezoidal rule was used for all numerical integration, due to its superior accuracy for periodic integrands. It was found that the solutions converged accurately for $N=51$ with 801 grid points for each integration.

\section{$5 \quad$ Results}

Some nonlinear solutions are now presented. The wavenumber was fixed at $k=1$, a value chosen with a view to obtaining nonlinear resonance effects. The linearized solution (as shown in Figure 2) indicates that such effects might be possible for two speeds at this wavenumber, namely the exchange flows at $F_{3} \approx-0.08$ and $F_{3} \approx-0.04$. These Froude numbers were allowed 


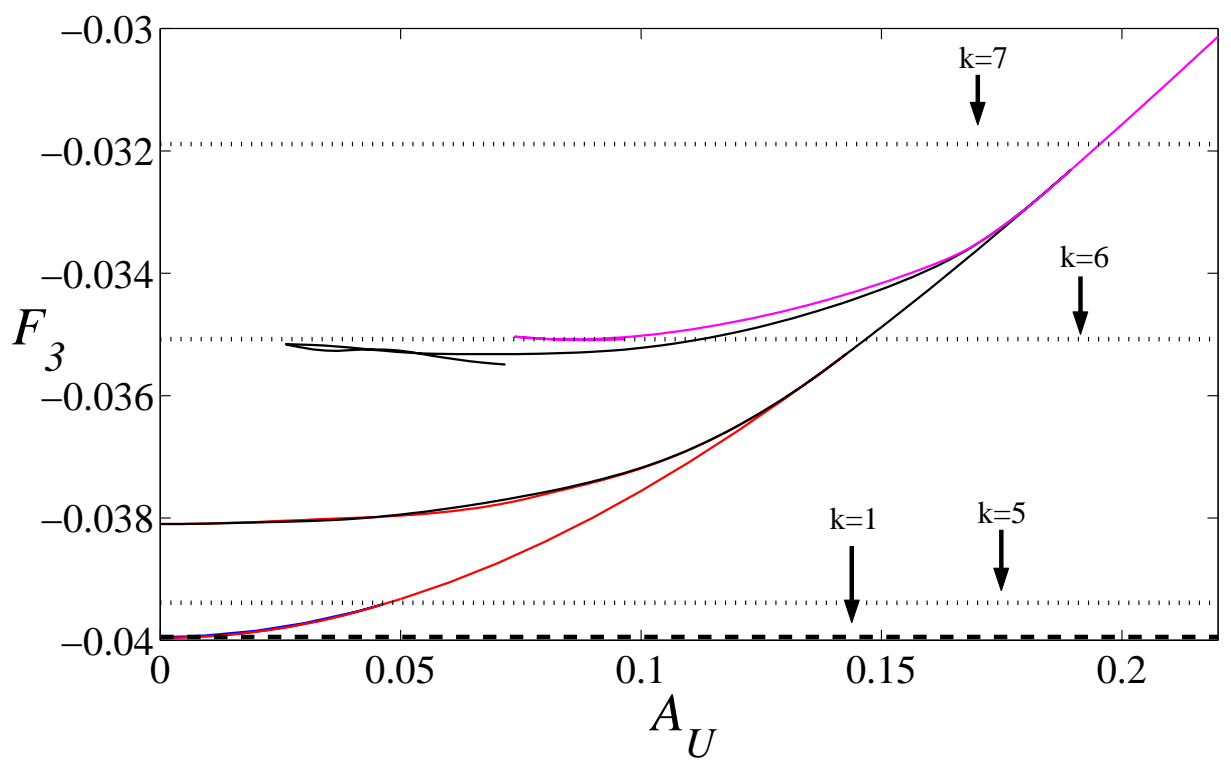

FiguRE 3: Dependence of lower layer Froude number $F_{3}$ on upper wave amplitude $\mathcal{A}_{U}$ for the slower speed case at $k=1$.

to vary as wave amplitude is increased, but the wavenumber and the remaining parameters were held constant at the same values as in the previously discussed linearized solution.

The first set of results is for the slower exchange flow, emerging from the linearized solution at $F_{3} \approx-0.04$. For small amplitudes this agrees very closely with the linearized solution and, as predicted, the interfaces are in phase. The relationship between the parameters $F_{3}$ and $\mathcal{A}_{U}$ is presented in Figure 3. Here the linearized solution is represented by a horizontal dashed line, with the series of multi-coloured solid lines representing various disjoint nonlinear solutions. Each of these solutions has a portion which traces out an increase in Froude number with increasing amplitude. In addition to this, at several points a pair of solutions bifurcates away from this main branch and continues through $\left(\mathcal{A}_{U}, F_{3}\right)$-space. These extra so- 


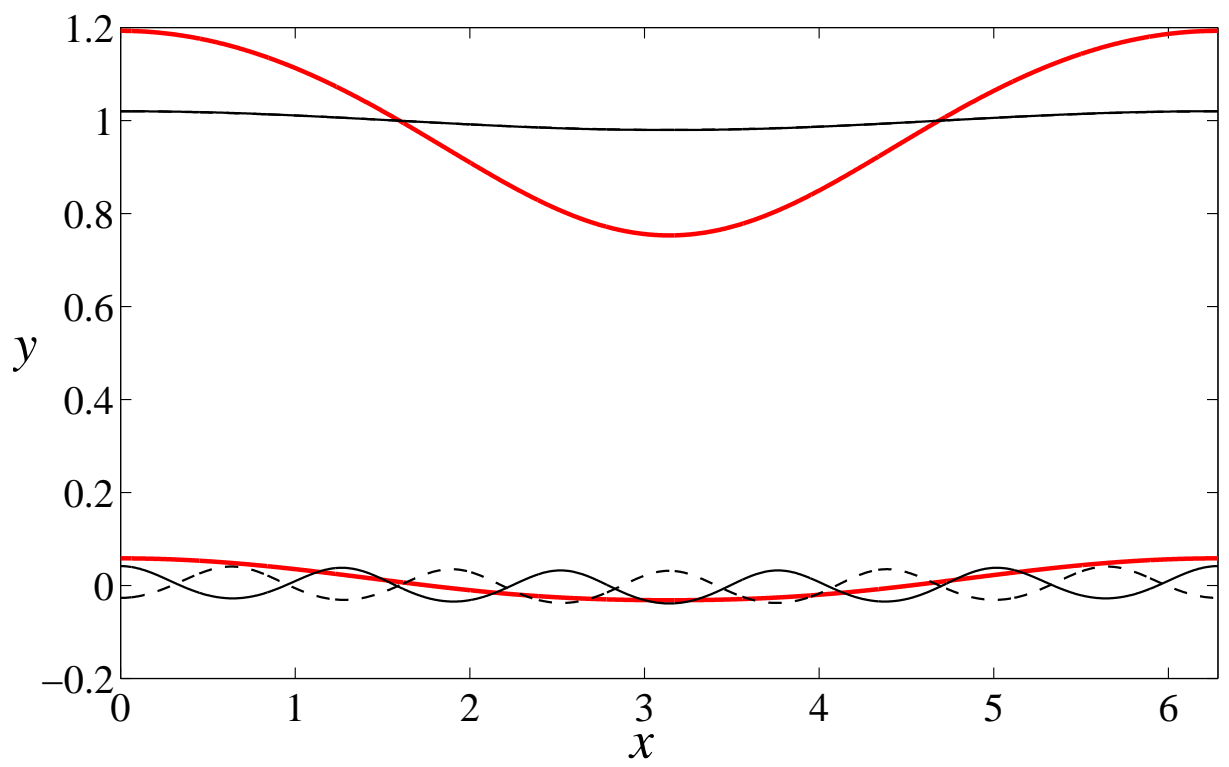

FiguRE 4: Three interfacial profiles for the slower speed case. The red lines are wave profiles for $\mathcal{A}_{U} \approx 0.21$. The solid and dashed black lines display a $1: 5$ resonance and are for $\mathcal{A}_{U} \approx 0.025$.

lutions display nonlinear resonant effects. The first set (bifurcating from $\left.\left(\mathcal{A}_{U}, F_{3}\right)=(0.0462,-0.0394)\right)$ is a $1: 5$ resonance which continues very close to the main branch, right back to $\mathcal{A}_{U}=0$ and beyond. These solutions have five ripples on the lower interface, that is, a superposed wave of shorter wavelength. This shorter wavelength component may be either in or out of phase with the primary component; hence the two branches.

The interfacial profiles for $\mathcal{A}_{U}=0.025$ are shown in Figure 4. Shown as a dotted line in Figure 3 is the linearized solution for $k=5$; this is the solution mode that interacts with the primary component of the wave to produce the resonance. Similarly the linearized solution for $k=6$ and $k=7$ are shown as dotted lines in Figure 3, with the solutions which bifurcate away these values of $F_{3}$ displaying $1: 6$ and $1: 7$ resonances, respectively. Many 


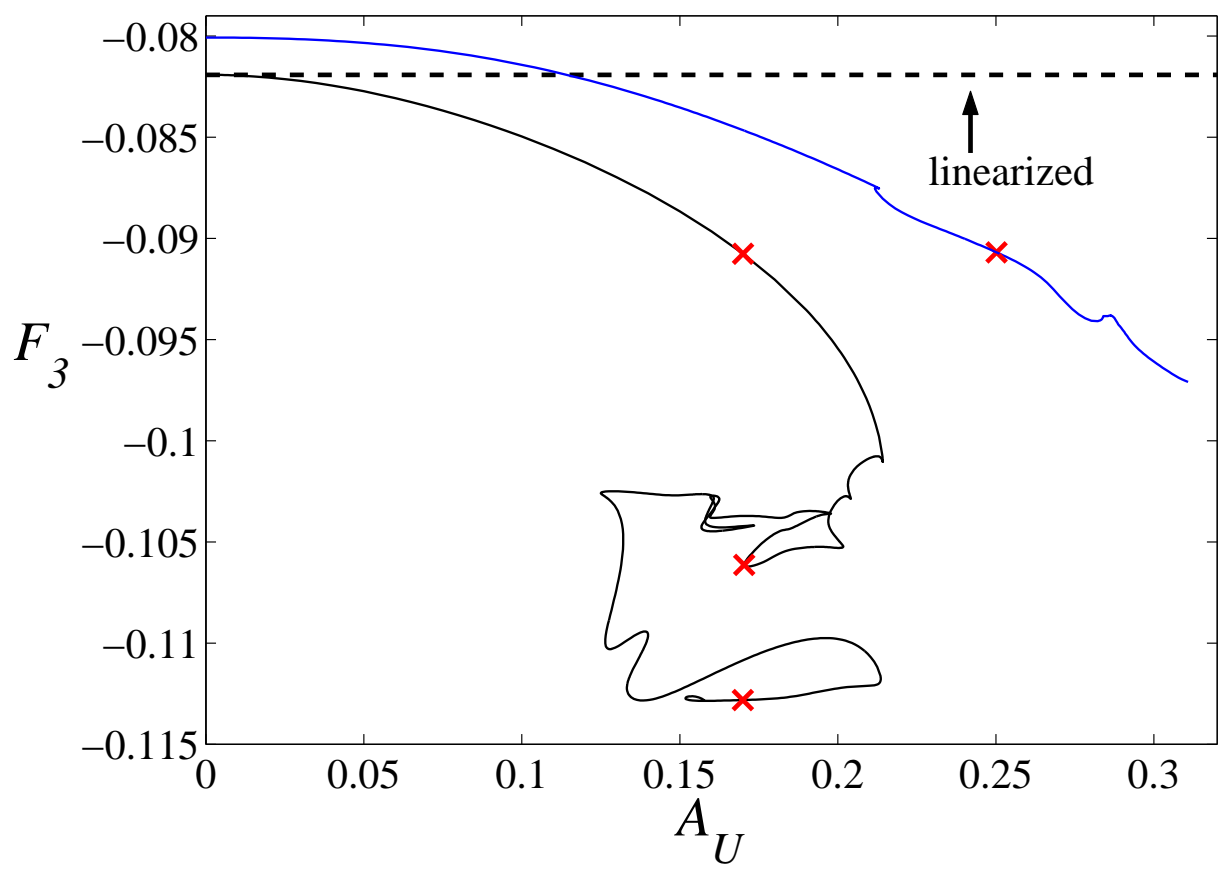

Figure 5: Dependence of lower layer Froude number $F_{3}$ on upper wave amplitude $\mathcal{A}_{U}$ for the faster speed case at $k=1$.

interfacial profiles have been computed along these solutions branches, but are not presented here in the interests of space; they typically possess many small waves on the lower interface (as in Figure 4). Each of these resonant effects is excited at a slightly faster speed than the linearized value of Froude number might suggest.

The second set of solutions at this wavenumber emerge from the linearized solution at $F_{3} \approx-0.08$. Again, at small amplitudes the nonlinear solutions agree closely with the linearized predictions. As amplitude is increased, the lower layer Froude number decreases until an amplitude of about $\mathcal{A}_{U} \approx 0.22$. Figure 5 shows the relationship between the lower fluid speed $F_{3}$ and the wave amplitude $\mathcal{A}_{U}$ for these two branches of solutions. The shapes of these 


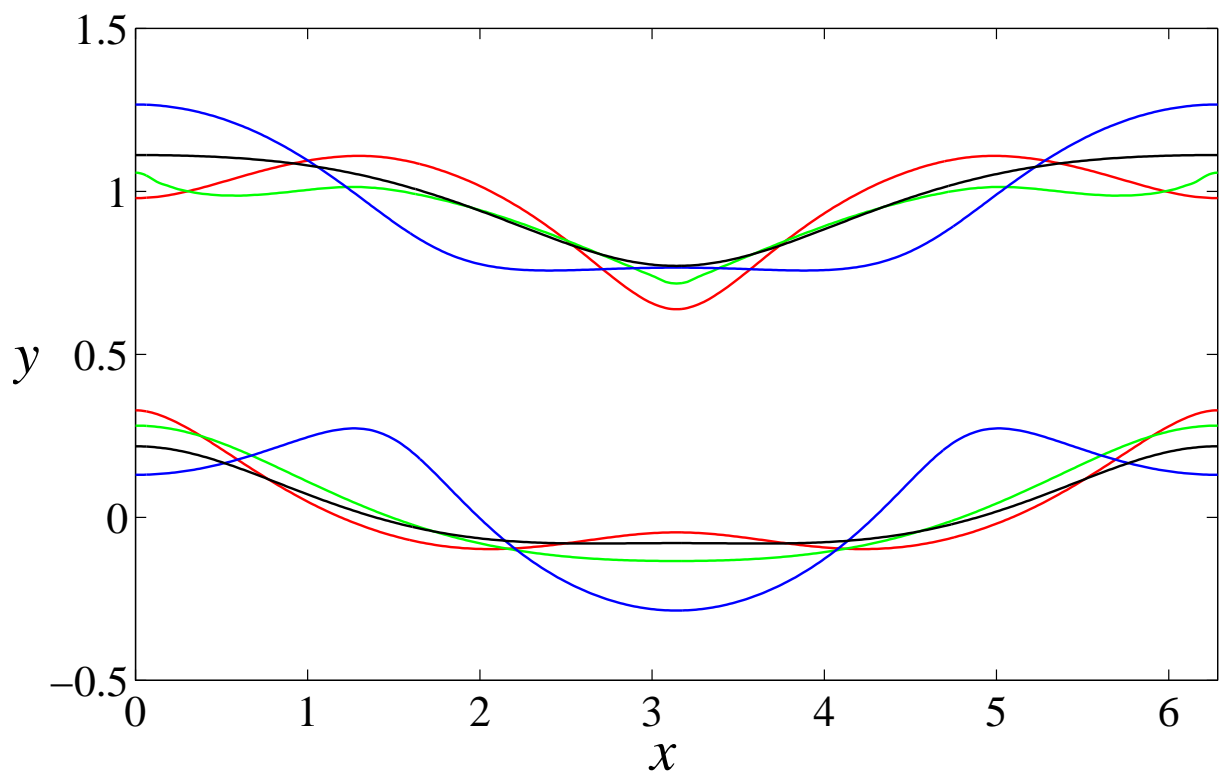

FiguRE 6: Four interfacial profiles for the faster speed case. The black, red and green wave profiles are all at an upper wave amplitude of $\mathcal{A}_{U} \approx 0.17$. The blue profiles are for $\mathcal{A}_{U} \approx 0.25$. All except the black profiles display a $1: 2$ resonance.

solution branches were found to be independent of the numerical parameters, and indicate the complex effects of non-linearity.

The solution profiles corresponding to the large crosses in Figure 5 are shown in Figure 6. A $1: 2$ resonance develops at around $\mathcal{A}_{U}=0.17$ (just after the first of the profiles shown) and persists through the solution space. As the solutions progress along this curve, the shorter wavelength component itself becomes of moderate amplitude and at least some of the fine structure in the $\left(\mathcal{A}_{U}, F_{3}\right)$ curve of Figure 5 may be attributed to this. There is a second set of solutions (shown with a blue line) where the short wavelength component is superposed with opposite phase to the solutions on the other branch. These 
may be traced right back to zero amplitude, giving a second small amplitude solution, while convergent solutions are unavailable for amplitudes greater than $\mathcal{A}_{U} \approx 0.31$. The lower layer Froude number of the small amplitude solution is near to, but slightly smaller than, the linearized solution for $k=2$.

\section{Conclusion}

A wide array of nonlinear solutions were computed, agreeing closely with the predictions of linear theory for small amplitudes. The focus of this work was to obtain nonlinear resonance effects between various solution modes, and these were found in abundance. Of particular interest were the excitation of successive resonances and the highly irregular relationship between the wave amplitude and lower layer speed parameters. Future work may consider the stability through time of the finite amplitude solutions.

\section{References}

[1] L. K. Forbes, G. C. Hocking, and D. E. Farrow. An intrusion layer in stationary incompressible fluids: Part 1: Periodic waves. Euro. Jnl of Applied Mathematics, 17:557-575, 2006. doi:10.1017/S0956792506006711. C52

[2] G. C. Hocking and L. K. Forbes. A note on the flow of a homogeneous intrusion into a two-layer fluid. Euro. Jnl of Applied Mathematics, 18:181-193, 2007. doi:10.1017/S0956792507006924. C52, C59

[3] H. Michallet and F. Dias. Non-linear resonance between short and long waves. Proc. of the 9th International Offshore and Polar Engineering Conference, pages 193-198, 1999.

http://cat.inist.fr/?aModele=afficheN\&cpsidt=1174804. C52 
[4] D. I. Pullin and R. H. J. Grimshaw. Interfacial progressive gravity waves in a two-layer shear flow. Phys. Fluids, 26:1731-1739, 1983. doi:10.1063/1.864372. C52, C59

[5] P. O. Rusås and J. Grue. Solitary waves and conjugate flows in a three-layer fluid. European Journal of Mechanics, B/Fluids, 21:185-206, 2002. doi:10.1016/S0997-7546(01)01163-3. C52, C59 


\section{Author addresses}

1. Michael J. Chen, School of Mathematics and Physics, University of Tasmania

mailto:mchen@utas.edu.au

2. Lawrence K. Forbes, School of Mathematics and Physics, University of Tasmania

mailto:Larry .Forbes@utas . edu . au 